\title{
Juegos, videojuegos y juegos serios: Análisis de los factores que favorecen la diversión del jugador
}

\author{
Dr. José Ramón Calvo-Ferrer |jr.calvo@ua.es \\ Universidad de Alicante
}

\author{
Palabras clave \\ juegos; videojuegos; diversión; motivación; \\ educación; formación; juegos serios \\ Sumario \\ 1. Introducción. 2. ¿Qué es el juego? 3. ¿Por \\ qué jugamos? 4. ¿Qué son los videojuegos? \\ 5. ¿Por qué nos atraen los videojuegos? 6. \\ ¿Qué características comparten los \\ videojuegos convencionales? 7. Videojuegos \\ ¿serios? 8. Conclusiones. 9. Bibliografía.
}

\section{Resumen}

A lo largo de la historia, numerosas teorías han tenido como objetivo explicar las razones que subyacen al ejercicio de la actividad lúdica. Con independencia de cualquier marco teórico, parece obvio que se juega a juegos y a videojuegos como consecuencia de que proporcionan diversión al jugador. Distintos autores sugieren que los videojuegos son óptimas herramientas educativas, desde la premisa de que atraen al jugador y favorecen el proceso de aprendizaje de diversas maneras. Como consecuencia de ello, educadores y docentes han favorecido el desarrollo e implementación de determinados videojuegos en el ámbito educativo conocidos como videojuegos serios con la esperanza de que faciliten la adquisición de contenidos académicos por parte del alumnado. Sin embargo, los videojuegos tradicionales poseen unas características particulares que, a diferencia de los videojuegos serios, les convierten en herramientas atractivas ante los ojos la mayor parte del público. El presente artículo tiene como objetivo poner de relieve los aspectos que hacen de los videojuegos herramientas divertidas, al tiempo que analiza las diferentes teorías que explican la actividad lúdica y propone una definición de juego y videojuego con el objeto de entender la peculiar idiosincrasia de los videojuegos serios.

\section{Cómo citar este texto:}

José Ramón Calvo-Ferrer (2018) Juegos, videojuegos y juegos serios: Análisis de los factores que favorecen la diversión del jugador. Miguel Hernández. Communication Journal, 9 (1), pp. 191 a 226. Universidad Miguel Hernández, UMH (Elche-Alicante).

DOI: http://dx.doi.org/10.21134/mhcj.v0i9.232 


\title{
Games, video games, and serious games: Analysing what makes playing fun
}

\author{
Dr. José Ramón Calvo-Ferrer |jr.calvo@ua.es \\ Universidad de Alicante
}

\begin{abstract}
Keywords
games; video games; fun; engagement; education; training; serious games

Summary

1. Introduction. 2. What are games? 3. Why do we play? 4 . What are video games? 5 . What makes video games engaging? 6 . Shared features in successful video games. 7. Serious games? 8. Concluding remarks. 9. References
\end{abstract}

\begin{abstract}
Throughout history, it has been often hypothesised on the reasons underlying game and play. Whatever the theoretical framework, it seems obvious that both games and video games are played upon the premise that they provide players with enjoyment and fun. Different authors have suggested that video games are optimal tools in education, since they engage players and trigger learning in different ways. In light of this, educators and policy-makers have seen an
\end{abstract} opportunity to devise and implement video games in education in hopes that they will infuse academic knowledge into students. However, traditional video games have specific traits that, unlike serious games, make them appealing and engaging to a vast majority of players. This paper attempts to highlight the aspects that make video games successfully entertaining by analysing the different theories that provide insight into the reasons why the human being plays. It also analyses the engagement-related core characteristics of play, and proposes a working definition of games and video games to highlight the idiosyncrasy of serious games.

\section{How to cite this text:}

José Ramón Calvo-Ferrer (2018) Games, video games, and serious games: Analysing what makes playing fun. Miguel Hernández Communication Journal, 9 (1), pp. 191 to 226. Universidad Miguel Hernández, UMH (Elche-Alicante).

DOI: http://dx.doi.org/10.21134/mhcj.v0i9.232 


\section{Introducción}

Una de las herramientas más populares en la actualidad son los videojuegos, plataformas ideadas para el entretenimiento que permiten en gran medida satisfacer las necesidades individuales en el plano del ocio, y que además ofrecen un enorme potencial para desarrollar acciones formativas, en opinión de numerosos autores, tanto en el ámbito académico como en el profesional. Si intentáramos averiguar las causas del éxito de los videojuegos, muy probablemente coincidiríamos en señalar la diversión inherente a éstos como el fundamento de su popularidad. Generalmente, la motivación principal que lleva a un individuo a jugar, ya sea por medio de videojuegos, juegos de mesa o actividades físicas, es la diversión que se obtiene durante el desarrollo de la actividad lúdica.

Numerosos estudios profundizan en los objetivos de la retórica clásica: docere, delectare et movere (enseñar, deleitar y emocionar) o, lo que es lo mismo, indican que un entorno que da cabida a la diversión del individuo favorece el aprendizaje. Desde este convencimiento, y con el objetivo específico de transmitir conocimientos entre los jugadores, surgen los conocidos «videojuegos serios», concebidos como herramientas para la educación y la formación, pero con unas características que, puesto que su objetivo principal no es provocar diversión en el jugador, les diferencia en gran medida de los videojuegos convencionales.

En línea con todo ello, es objeto del presente trabajo analizar e identificar los paralelismos existentes entre el concepto de juego y el desarrollo de la actividad lúdica a través de los videojuegos, examinando detenidamente las posibles similitudes y diferencias entre uno y otro que permita llevar a cabo un análisis de los denominados «videojuegos serios», que en ocasiones parecen ideados tan sólo como un soporte en el que poder introducir contenidos docentes, obviando tanto su tradicional carácter motivador como su capacidad para generar entretenimiento. 


\section{2. ¿Qué es el juego?}

Resulta curioso comprobar cómo en numerosas ocasiones, cuanto más consabido es un elemento o mayor es su tradición en la sociedad, mayor discrepancia genera en torno a su definición. En esta línea, son numerosas las ocasiones en que se ha intentado ofrecer una definición de la actividad lúdica, bien en consonancia con los principios de un marco teórico concreto, bien centrándose en distintos aspectos de su propia realidad. En cualquier caso, no parece desacertado afirmar que el juego posee unas características complejas que dificultan tanto su definición como su categorización, tal como se deduce de las distintas opiniones que se muestran a continuación.

En primer lugar, cabe mencionar que en los distintos planteamientos teóricos sobre el juego se observan principalmente tres aspectos sobre los que se articula su definición (cf. Juul, 2003): forma y estructura, vínculo entre juego y jugador y, por último, relación entre juego y otros aspectos externos al mismo. De este modo, entendiendo el juego como sistema formal, el concepto de norma constituye un elemento básico para considerar la actividad lúdica como tal, como se puede comprobar en las definiciones de Suits (1976), Kelley (1988) y Salen y Zimmerman (2006):

To play a game is to engage in activity directed towards bringing about a specific state of affairs, using only means permitted by rules, where the rules prohibit more efficient in favor of less efficient means, and where such rules are accepted just because they make possible such activity. (Suits, 1976: 34)

A game is a form of recreation constituted by a set of rules that specify an object to be attained and the permissible means of attaining it. (Kelley, 1988: $50)$

A game is a system in which players engage in an artificial conflict, defined by rules, that results in a quantifiable outcome. (Salen y Zimmerman, 2006: 96) 
Por otra parte, Avedon y Sutton-Smith (1981: 7) parten del vínculo que se establece entre juego y jugador, haciendo especial hincapié en factores como los objetivos o los distintos tipos de interacción que se origina en el seno de ese sistema de normas:

At its most elementary level then we can define game as an exercise of voluntary control systems in which there is an opposition between forces, confined by a procedure and rules in order to produce a disequilibrial outcome.

Del mismo modo, otras definiciones parten de la relación existente entre el juego y otros aspectos externos al mismo y a los sujetos que toman parte en él. De este modo, mientras Crawford (1982) considera el juego esencialmente conflicto e interactividad, Huizinga (1949: 13) afirma que se trata de una actividad voluntaria que reporta satisfacción al jugador por su carácter informal y que además favorece la formación de grupos:

Summing up the formal characteristics of play we might call it a free activity standing quite consciously outside "ordinary" life as being "not serious", but at the same time absorbing the player intensely and utterly. it is an activity connected with no material interest, and no profit can be gained by it. It proceeds within its own proper boundaries of time and space according to fixed rules and in an orderly manner. It promotes the formation of social groupings which tend to surround themselves with secrecy and to stress their difference from the common world by disguise or other means.

Como se puede observar, la mayoría de estas definiciones ofrecen más puntos en común que opiniones enfrentadas. Principalmente, ello obedece al hecho de que no se formulan atendiendo a idénticos aspectos del juego, sino que ofrecen puntos de vista distintos de una misma realidad. 
En esta línea, Crawford (1982: 7) señala que todos los juegos poseen cuatro características comunes: «representation, interaction, conflict, and safety». En primer lugar, señala, el juego crea una visión subjetiva simplificada de la realidad en representación de la misma. Por otra parte, la interacción convierte el mero ejercicio técnico en juego, puesto que incorpora un elemento social o interpersonal que convierte la actividad en conflicto que, bien de forma directa o indirecta, también es parte intrínseca del juego. Por último, afirma que el juego permite que este ejercicio se lleve a cabo dentro de un contexto seguro.

Callois (2006: 128), por su parte, define el juego ofreciendo una recapitulación de las características formales del mismo, señalando que se trata de una actividad libre, independiente, incierta, improductiva, regida por normas e imaginaria:

1. Free: in which playing is not obligatory; if it were, it would at once lose its attractive and joyous quality as diversion.

2. Separate: circumscribed within limits of space and time, defined and fixed in advance.

3. Uncertain: the course of which cannot be determined, nor the result attained beforehand, and some latitude for innovations being left to the player's initiative.

4. Unproductive: creating neither goods, nor wealth, nor new elements of any kind; and, except for the exchange of property among the player, ending in a situation identical to that prevailing at the beginning of the game.

5. Governed by rules: under conventions that suspend ordinary laws, and for the moment establish new legislation, which alone counts.

6. Make-believe: accompanied by a special awareness of a second reality or of a free unreality, as against real life.

Teniendo todo ello en cuenta, parecen factores clave en la actividad lúdica su modo de ejecución, su estructura y el vínculo que establece con el jugador. Por 
tanto, en consonancia con las definiciones anteriores, parece oportuno entender y definir el juego como «ejercicio lúdico delimitado por normas ejercido de forma voluntaria».

\section{3. ¿Por qué jugamos?}

Si numerosas son las definiciones de juego, no le van a la zaga las teorías que pretenden explicar el porqué de esta actividad ${ }^{1}$. De este modo, son muchos los autores que han teorizado sobre los motivos que nos impulsan a jugar. A continuación se presentan las más ilustrativas, amalgamando principalmente la categorización de Gutiérrez (2004), que distingue entre teorías biológicas, psicológicas, antropológicas y socioculturales, y evolutivas, con las propuestas y aclaraciones que ofrecen tanto Romero y Gómez (2008) como Montañés et al. (2000).

\subsection{Teońas biológicas}

Las teorías biológicas consideran el juego como una actividad necesaria para la supervivencia y para restablecer el equilibrio del organismo, bien para liberar sobrantes de energía, bien para obtenerla en periodos de agotamiento. En este marco, encontramos las siguientes propuestas teóricas:

Teoría de las necesidades o de la potencia superflua. De acuerdo con Schiller (1795), el ser humano elimina por medio del juego la energía sobrante tras cubrir las necesidades biológicas básicas.

1 Véase Gruppe (1976; en Gutiérrez Delgado, 2004: 158): «El juego se resiste a una clasificación conceptual definitiva, ya que cualquier teoría, lo más, logra, si no quiere sobrepasar sus propios planteamientos, es captar una parte de la verdad del juego». 
Teoría del exceso de energía. En línea con lo expresado por Schiller, Spencer (1897; en Evans y Pellegrini, 1997) expone la idea de que el juego es un mecanismo a través del cual se eliminan las energías sobrantes. Partiendo de la concepción de que los seres vivos poseen una cantidad de energía para consumir a lo largo de la jornada, establece que aquellas especies que no la consumen en su totalidad para cubrir las necesidades primarias la pueden utilizar en otras actividades, entre ellas, el juego.

Teoría de la terapia de restablecimiento. Contrariamente a lo expresado por Schiller y por Spencer, Lazarus (1883) afirma que por medio del juego los seres vivos descansan de otras actividades que producen fatiga. Por tanto, el juego no supone un gasto de energía, sino que se trata de un mecanismo para proporcionar relajación y energía al individuo en momentos de fatiga.

El juego como anticipación funcional. Por su parte, Groos (1902; en Martínez Rodríguez, 2008) afirma que el juego es un procedimiento instintivo a través del cual el individuo practica y adquiere comportamientos que deberá utilizar en situaciones futuras, necesarios para su supervivencia.

Teoría de la derivación por ficción. Para Claparède (1927; en Tabernero del Río, 1997), el juego tiene una gran importancia en la vida del niño. Siguiendo la propuesta teórica de Groos, afirma que las nociones de obligación moral, de deber, de necesidad social, de necesidad material, ausentes en el niño frente al adulto, están sustituidos por el juego. En esta línea, se considera como una actitud del organismo ante la realidad y cuya clave es la ficción.

\subsection{Teorías psicológicas}

Por otra parte, las teorías psicológicas tratan de conceptualizar la actividad lúdica estudiando la relación que se establece entre el juego y la personalidad. Para ello, tiene en cuenta aquellos aspectos característicos del juego a lo largo de las distintas etapas del desarrollo del individuo con el objeto de poder 
entender en profundidad cuáles son las implicaciones psicológicas derivadas de la actividad.

Teoría del juego infantil. Freud (1920) establece que el juego es la manifestación de tendencias o deseos reprimidos y ocultos y surge de la necesidad de satisfacerlos, y de expresar y comunicar sus experiencias y las emociones que las acompañan. En su opinión, el juego es un mecanismo del ser humano para resolver conflictos por medio de la ficción.

El juego como asimilación de la realidad. Piaget (1945) destaca la importancia del juego en los procesos de desarrollo, relacionando el desarrollo de los estadios cognitivos con el desarrollo de la actividad lúdica. En otras palabras, las transformaciones que se producen en las estructuras cognitivas del niño modelan las diversas formas de juego que surgen a lo largo del desarrollo infantil, lo que supone un ejercicio de adaptación al medio y de asimilación de la realidad.

\subsection{Teońas antropológicas y socioculturales}

A diferencia de las teorías anteriores, las explicaciones antropológicas y socioculturales permiten plantear el juego como instrumento para la educación, pues le confieren una importancia capital en el desarrollo cognitivo del niño.

Teoría del juego protagonizado. Vygotsky (1933; en Robbins, 2001) entiende el juego como una guía del desarrollo del niño, en cuanto crea situaciones imaginarias que operan como sistemas de apoyo mental. El juego se desarrolla desde un plano conceptual, estableciendo situaciones imaginarias que obligan al niño a definirse en sus actos, procediendo fuera de la percepción directa. En esta línea, Elkonin (1978; en Garaigordobil Landazábal, 2007) añade que el fondo, la naturaleza y el origen del juego es social, y que nace de las condiciones de vida del niño en la sociedad. 


\subsection{Teorías evolutivas}

Por su parte, las teorías evolutivas establecen el juego como herramienta indispensable para el desarrollo evolutivo del individuo.

- La teoría de la recapitulación. Hall (1906) afirma que por medio del juego el ser humano revive las actividades de otras generaciones y la evolución de la cultura humana, reproduciéndola a través de la actividad lúdica. De este modo, el juego tiene un origen biológico cuya función es la de liberar al ser humano de residuos ancestrales e impulsar al desarrollo de otras actividades que favorezcan la evolución.

- Teoría de la dinámica infantil. Buytendijk (1933) afirma que el juego se explica desde la propia infancia, es decir, que el ser vivo juega porque es joven, y determina una serie de características de la infancia que llevan al individuo a jugar: ambigüedad, carácter impulsivo y actitud patética ante la realidad y la timidez.

- Teoría del juego como afirmación placentera. Chateau (1973; en Gutiérrez Delgado, 2004) asegura que el juego es el único medio que tiene el niño para autoafirmarse. Creando dificultades, riesgos y reglas, el niño experimenta un placer moral al superarlas, al tiempo que desarrolla su capacidad intelectual. En esta línea, asegura, se desarrollan las funciones latentes, comprendiendo que el individuo más capacitado es también aquel que juega mejor.

- Teoría del juego y el espacio potencial. Winnicott (1982) afirma que el juego se desarrolla en el espacio potencial, que es la zona que se encuentra entre el niño y la madre. En este espacio es donde se originan los objetos transicionales (un chupete, un peluche, etc.), cuya función es la de suplir determinadas funciones de la madre cuando ésta está ausente. De este modo, el niño establece un espacio intermedio de seguridad entre él mismo y la realidad, constituyendo un escenario lúdico a través de las relaciones establecidas con el objeto transicional en cuestión. 
Como se puede observar, son numerosas las hipótesis que intentan explicar desde una perspectiva teórica el concepto de juego, tanto en lo relativo a su definición como a los motivos conducentes al propio ejercicio de la actividad lúdica. Una de sus formas, los videojuegos, dan igualmente cuenta de la ambigüedad del concepto y de la dificultad de cualquier pretendida taxonomización al respecto.

\section{4. ¿Qué son los videojuegos?}

Tal como sucede con el juego, el concepto de videojuego atiende a numerosas explicaciones. Del análisis detallado de las distintas definiciones propuestas, observamos cierta similitud entre los puntos de vista desde los que se definen el concepto de juego y aquellos que intentan explicar qué son los videojuegos.

De este modo, Lin y Lepper (1987) hacen especial hincapié en tres elementos distintos: componente tecnológico, tipo de videojuego y soporte en que se juega. Levis (2005: 27), por su parte, define los videojuegos como «un entorno informático que reproduce sobre una pantalla un juego cuyas reglas han sido previamente programadas» con lo que, al igual que autores como Suits (1976), Kelley (1988), Salen y Zimmerman (2004, 2006), o Salen, Zimmerman y Goldstein (2005), hace referencia a los videojuegos como un sistema formal, poniendo de relieve las normas que lo rigen.

Pelegrina y Tejeiro (2003) y, especialmente, Marquès (2000), señalan el vínculo interactivo que se genera entre videojuego y jugador, en línea con lo expresado por Crawford (1982):

[...] todo juego electrónico con objetivos esencialmente lúdicos, que se sirve de la tecnología informática y permite la interacción a tiempo real del jugador con la máquina, y en el 
que la acción se desarrolla fundamentalmente sobre un soporte visual (que puede ser la pantalla de una consola, de un ordenador personal, de un televisor, o cualquier otro soporte semejante). (Pelegrina del Río y Tejeiro Salguero, 2003: 20)

Entendemos por videojuegos todo tipo de juego digital interactivo, con independencia de su soporte (ROM interno, cartucho, disco magnético u óptico, on-line) y plataforma tecnológica (máquina de bolsillo, videoconsola conectable al TV, teléfono móvil, máquina recreativa, microordenador, ordenador de mano, vídeo interactivo). (Marquès, 2000)

Provenzo (1991) hace hincapié en el poder de los videojuegos como una puerta abierta por la sociedad de consumo, que permite conectarnos con enormes y poderosas redes de información, comunicación, ocio y entretenimiento, enfatizando la relación existente entre el juego y otros aspectos externos al mismo y a los sujetos que toman parte en él, mientras que Frasca (2001) y Rodríguez (2002), en similitud con Huizinga (1949), ponen de manifiesto su carácter lúdico y la posibilidad de interacción entre jugadores:

[...] any forms of computer-based entertainment software, either textual or image-based, using any electronic platform such as personal computers or consoles and involving one or multiple players in a physical or networked environment. (Frasca, 2001: 4)

[...] entenderemos por videojuego todo juego electrónico con objetivos esencialmente lúdicos que sirviéndose de la tecnología informática puede presentarse en distintos soportes (fundamentalmente consolas y ordenadores). (Rodríguez, 2002: 16) 
¿Cuál es, por tanto, la diferencia entre juego y videojuego? Si se analiza en detalle las definiciones propuestas anteriormente, el elemento diferenciador entre ambos parece exclusivamente limitado a la dependencia de este último con la tecnología o, dicho con otras palabras, el soporte en que se desarrolla. Kirriemuir (2002) define los videojuegos como juegos que cuentan con las siguientes seis características formales:

1. playable using a television set. The game software is accessed via a games console, to which input devices such as joysticks or controllers are attached; or 2. playable using a television set, with the game being accessible or downloadable through a satellite or digital subscription-based system; or

3. playable on a PC or Macintosh; or

4. housed inside a cabinet with a built-in screen and input device such as a joystick. These are typically found in arcades; or

5. found on small, portable games machines, of which the most well-known is the Game Boy; or

6. increasingly found in consumer electronic devices, such as mobile phones and handheld PCs.

Por su parte, más recientemente, García Gigante (2009: 19) define los videojuegos como «un tipo especial de juego $[. .$.$] que para su uso requiere de$ algún medio o tipo de soporte electrónico, y que suele contar con distintos periféricos para conectarse a él, que [...] se caracteriza por permitir la interacción entre el videojuego y uno o varios jugadores [...], que dicha interacción es consecuencia de la información que el usuario recibe del medio y que puede ser visual, auditiva [...] o táctil [...]».

De la definición de Kirriemuir (2002) extraemos que su propuesta no ofrece en realidad detalle sobre las características de los videojuegos, sino que establece una clasificación de los mismos de acuerdo con el soporte desde el que se ejecutan (videoconsola, servidor, ordenador, dispositivo móvil, etc.). 
Por otra parte, la definición de García Gigante (2009), surgida a partir del estudio y análisis de otras definiciones, parece circunscrita en exceso a la coyuntura temporal desde la que se formula, siendo la afirmación de que «para su uso requiere de algún medio o tipo de soporte electrónico» el elemento de mayor permanencia temporal ${ }^{2}$.

¿Significa todo ello, por tanto, que la única diferencia entre juego y videojuego radica en el soporte electrónico de los últimos? Muy probablemente no, aunque tanto juego como videojuego poseen unas características propias derivadas del medio en que se desarrollan. De este modo, se podría afirmar que los videojuegos tienen efectos distintos a los de los juegos tradicionales en el desarrollo de la coordinación óculo-manual del jugador, o que los juegos no precisan de elementos externos al mismo para ser jugados, pero no dejarían de ser apreciaciones estrechamente ligadas con la presencia o ausencia del mencionado componente tecnológico.

Por tanto, partiendo de la definición de juego propuesta anteriormente, entendemos y definimos el videojuego como un «ejercicio lúdico delimitado por normas ejercido de forma voluntaria a través de un hardware específico». No obstante, estamos seguros de que muy probablemente aparecerán numerosos videojuegos que en un futuro cuestionarán la validez de esta definición, por amplia y genérica que pueda resultar en la actualidad. Por tanto, quizá sería adecuado hacerse a la idea de la volatilidad de determinadas definiciones en ciertos ámbitos o asumir, como explica Tavinor (2009: 2), que «a long history of failed definitions in a particular case might convince us that a definition in that instance is futile».

${ }^{2} \mathrm{Si}$ atendemos a las variaciones y modificaciones a las que se encuentran sujetos los videojuegos en materia tecnológica, parece oportuno prescindir de todo detalle susceptible de cambio o evolución, como es el caso de los elementos que posibilitan la interacción entre jugador y máquina, o entre los propios jugadores, y los modos y formas en los que ésta se produce. 
Digresiones terminológicas aparte, huelga decir que los videojuegos gozan de amplia popularidad y difusión en nuestra sociedad desde su aparición, que se remonta a mediados del siglo XX, como consecuencia, entre otras razones, de su evidente atractivo (Méndez González \& Calvo-Ferrer, 2017).

\section{5. ¿Por qué nos atraen los videojuegos?}

Malone (1981: 333) señala que los videojuegos logran captar y mantener la atención como consecuencia de la motivación que despiertan en el jugador. Esta motivación, que califica como intrínseca al propio juego, se fundamenta a su vez en tres aspectos fundamentales, «challenge, fantasy, and curiosity»:

Challenge is hypothesized to depend on goals with uncertain outcomes. Several ways of making outcomes uncertain are discussed, including variable difficulty level, multiple level goals, hidden information, and randomness. Fantasy is claimed to have both cognitive and emotional advantages in designing instructional environments. A distinction is made between extrinsic fantasies that depend only weakly on the skill used in a game, and intrinsic fantasies that are intimately related to the use of the skill. Curiosity is separated into sensory and cognitive components, and it is suggested that cognitive curiosity can be aroused by making learners believe their knowledge structures are incomplete, inconsistent, or unparsimonious.

Del mismo modo, Malone y Lepper (1987), identifican dos tipos de motivaciones: individuales e interpersonales. En la primera de ellas añaden el concepto de «control» a los mencionados anteriormente: «challenge, fantasy, and curiosity». Por otra parte, los factores que incluyen en el tipo de motivación interpersonal son «cooperación», «competencia» y «reconocimiento»: 
Tabla 1. Ficha: Motivations, 1987.

\begin{tabular}{|c|c|c|}
\hline \multirow[t]{10}{*}{$\begin{array}{l}\text { INDIVIDUAL } \\
\text { MOTIVATIONS }\end{array}$} & \multirow[t]{4}{*}{ Challenge } & $\begin{array}{l}\text { Goals: (a) clear, fixed goals; or (b) ability for } \\
\text { learners to generate goals for themselves. }\end{array}$ \\
\hline & & $\begin{array}{l}\text { Uncertain Outcomes: (a) variable difficulty; (b) } \\
\text { multiple levels of goals; (c) hidden information, } \\
\text { selectively revealed; and (d) randomness. }\end{array}$ \\
\hline & & $\begin{array}{l}\text { Performance Feedback: frequent, clear, } \\
\text { constructive, and encouraging. }\end{array}$ \\
\hline & & $\begin{array}{l}\text { Self-Esteem: (a) gradually increasing difficulty levels } \\
\text { to promote feelings of competence; (b) goals that } \\
\text { are meaningful to the learner. }\end{array}$ \\
\hline & \multirow[t]{2}{*}{ Curiosity } & $\begin{array}{l}\text { Sensory Curiosity: may be promoted using variable } \\
\text { audio \& visual effects. }\end{array}$ \\
\hline & & $\begin{array}{l}\text { Cognitive Curiosity: may be promoted by: (a) using } \\
\text { surprise, paradoxes, incompleteness; and (b) using } \\
\text { activities that contain topics in which the learner is } \\
\text { already interested. }\end{array}$ \\
\hline & \multirow[t]{3}{*}{ Control } & $\begin{array}{l}\text { Contingency: learning environment should be } \\
\text { responsive to learner actions. }\end{array}$ \\
\hline & & $\begin{array}{l}\text { Choice: activities should provide learner with choice } \\
\text { over various aspects of the learning environment } \\
\text { (e.g., narration or full text). }\end{array}$ \\
\hline & & $\begin{array}{l}\text { Power: activity should allow learner to produce } \\
\text { powerful effects. }\end{array}$ \\
\hline & Fantasy & $\begin{array}{l}\text { Emotional aspects: appeal to the emotional needs } \\
\text { of learners. }\end{array}$ \\
\hline
\end{tabular}




\begin{tabular}{|c|c|}
\hline & $\begin{array}{l}\text { Cognitive Aspects: use appropriate metaphors or } \\
\text { analogies for the material to be learned. }\end{array}$ \\
\hline & $\begin{array}{l}\text { Endogeneity: fantasies should have an integral } \\
\text { (endogenous) relationship to the material to be } \\
\text { learned. }\end{array}$ \\
\hline \multirow{3}{*}{$\begin{array}{l}\text { INTERPERSONA } \\
\text { L } \\
\text { MOTIVATIONS }\end{array}$} & $\begin{array}{l}\text { Cooperation: design some activities to promote cooperation } \\
\text { among learners. }\end{array}$ \\
\hline & $\begin{array}{l}\text { Competition: design some activities to require learners to } \\
\text { compete with one another (e.g., actions affect each other). }\end{array}$ \\
\hline & $\begin{array}{l}\text { Recognition: learners' efforts should receive social recognition } \\
\text { so they are appreciated by other. }\end{array}$ \\
\hline
\end{tabular}

Fuente: Malone y Lepper (1987).

En esta línea, Amory, Vincent y Adams (1998; en Kirriemuir y McFarlane, 2004) señalan que la curiosidad es un motivo fundamental que hace que los videojuegos sean atractivos. Por su parte, Kirriemuir y McFarlane (2004) apuntan que, si bien la curiosidad motiva al jugador, es la calidad de lo que sucede en el videojuego el factor que hace que se mantenga su atención e interés.

Marquès (2000) incide también en la cuestión de la dificultad como elemento clave para captar la atención del jugador. En su opinión, «los videojuegos representan un reto continuo para los usuarios, que deben construir y aplicar estrategias cognitivas y desarrollar determinadas habilidades psicomotrices para afrontar las situaciones problemáticas que se van sucediendo ante la pantalla», lo que hace que el jugador siempre se implique y se vea «obligado a tomar decisiones y ejecutar acciones motoras continuamente». 
De todos modos, en conjunción con el nivel de dificultad de los videojuegos y el reto que éstos puedan plantear al jugador, Marquès afirma que «con más o menos tiempo y esfuerzo siempre se suele lograr el objetivo que se pretende, ya que no se requieren ni grandes conocimientos previos específicos ni una especial capacidad intelectual», hecho que también favorece la implicación del jugador.

Por su parte, Cortés (2010: 32) establece como elemento clave en el atractivo de los videojuegos la interacción que éstos ofrecen al jugador frente a otras formas de ocio:

La interacción con el videojuego se convertirá, de este modo, en la característica más destacada de estos medios y que los hace tan atractivos. Contrastan en este punto con la contemplación de la televisión o el cine que, aunque mantengan intelectualmente estimulados y activen la imaginación, dejan poca iniciativa a la persona que los ve. Los videojuegos exigen una implicación activa en el propio desarrollo de los mismos y el jugador se ve obligado a tomar decisiones que le involucran en el proceso del juego.

De acuerdo con Prensky (Prensky, 2001), los videojuegos logran atraer la atención no sólo porque son divertidos, sino porque poseen otras muchas virtudes. Principalmente, explica, el hecho de que sean divertidos proporciona placer y satisfacción al jugador. Así mismo, indica, son atractivos porque obedecen a reglas, favoreciendo la aparición de estructuras lógicas, y porque están basados en objetivos, lo que aumenta la motivación del jugador. También destaca el hecho de que ofrezcan un feedback constante y sean adaptativos de acuerdo con los conocimientos del jugador, lo que favorece el aprendizaje.

Del mismo modo, indica que el hecho de que los videojuegos ofrezcan recompensas atrae al jugador aumentando su ego, y asegura que, dado que presentan conflictos, competición, retos y requieren solucionar problemas, 
aumentan además la creatividad. Finalmente, el hecho de que los videojuegos ofrezcan emoción, puesto que se presentan enmarcados en una narrativa, o que sean interactivos y permitan interactuar socialmente, constituyen, según Prensky (2001: 05-1), factores que determinan el alto grado de atracción que ejercen sobre el jugador:

1. Games are a form of fun. That gives us enjoyment and pleasure.

2. Games are form of play. That gives us intense and passionate involvement.

3. Games have rules. That gives us structure.

4. Games have goals. That gives us motivation.

5. Games are interactive. That gives us doing.

6. Games are adaptive. That gives us flow.

7. Games have outcomes and feedback. That gives us learning.

8. Games have win states. That gives us ego gratification.

9. Games have conflict/competition/challenge/opposition. That gives us adrenaline.

10. Games have problem solving. That sparks our creativity.

11. Games have interaction. That gives us social groups.

12. Games have representation and story. That gives us emotion.

Por su parte, Koster (2005) sitúa el aprendizaje como causante, y no como consecuencia, de la diversión: en su opinión, el cerebro humano crea asociaciones neuronales para afianzar patrones que previamente ha identificado. La predicción cerebral de patrones conocidos refuerza estas conexiones neuronales, produciendo de este modo una retroalimentación positiva mediante endorfinas y dopaminas, y generando una sensación de diversión en el individuo. En esta línea, Gee (2005: 15) realiza la siguiente reflexión: 
When we think of games, we think of fun. When we think of learning we think of work. Games show us this is wrong. They trigger deep learning that is itself part and parcel of the fun. It is what makes good games deep.

Del mismo modo, Gallego y Llorens (2011) afirman que practicar no es más que repetir situaciones para poner a prueba los circuitos neuronales de reconocimiento de patrones, reforzándolos mediante el ejercicio para conseguir reconocerlos de forma más rápida y con un menor número de errores. Por tanto, para estos autores, el propio aprendizaje es causante de la diversión:

La diversión es una recompensa por mejorar nuestra capacidad de reconocer patrones, es decir, es una recompensa por aprender. [...] La diversión no es un catalizador del aprendizaje, sino que es la consecuencia del propio aprendizaje. Por tanto, cuando nos divertimos es porque estamos aprendiendo. [...] Los videojuegos son todos educativos en sí mismos. Puesto que la diversión es aprendizaje, si jugamos para divertirnos, jugamos para aprender. Por tanto, lo que nos atrae de los videojuegos es que nos permiten practicar.

El informe Video Gamers in Europe, mencionado en páginas anteriores, ofrece una interesante distinción entre las motivaciones de los usuarios de videojuegos en general y aquellas de quienes juegan en dispositivos portátiles. De este modo, según indica el estudio, los encuestados hacen uso de videojuegos, principalmente, porque les ofrecen diversión (61\%), porque les permiten descansar de otras actividades (53\%) y porque consideran que se trata de una buena manera de pasar el tiempo (53\%). Específicamente en relación con los dispositivos portátiles, el estudio afirma que la razón principal esgrimida como motivo para su uso por parte del $52 \%$ de los encuestados es «cuando me aburro/para hacer tiempo».

Una encuesta de la Interactive Digital Software Association (2001) ofrece cuatro razones fundamentales por las que los videojuegos resultan atractivos. 
Un $87 \%$ de los jugadores encuestados respondió que juega a videojuegos porque son divertidos. Del mismo modo, un 72\% afirmó que lo hace también porque, por su dificultad, suponen un reto y un desafío. Por otra parte, el $42 \%$ de los encuestados señaló además que los juegos le resultan atractivos porque son una forma de interacción social que se puede compartir con familia y amigos, mientras que el 36\% afirmó que ofrecen mucho entretenimiento en comparación con el dinero que cuestan ${ }^{3}$.

Parece evidente, por tanto, que a ojos del individuo la diversión es un factor clave en el empleo de videojuegos. Pero, ¿qué características comparten estas herramientas que hacen percibir al usuario una sensación de diversión durante su empleo?

\section{6. ¿Qué características comparten los videojuegos convencionales?}

De igual manera que a la hora de identificar los factores por los que los videojuegos resultan atractivos, existen diversas opiniones en torno a qué características comparten los videojuegos convencionales, entendidos como aquellos que logran captar y mantener la atención del jugador o, dicho de otra manera, aquellos que proporcionan diversión y entretenimiento. No obstante, como se observa a continuación, muchos de los conceptos presentados no son excluyentes entre sí, sino que obedecen, según nuestro entender, a distintas

\footnotetext{
${ }^{3}$ Analizando de forma pormenorizada los datos recogidos por la encuesta, se observa cierta correspondencia entre las teorías expuestas en páginas anteriores y los motivos esgrimidos por los encuestados. De este modo, la importancia del componente lúdico planteada por Huizinga (1949) se correspondería con el dato de que un $87 \%$ de los encuestados juega a videojuegos porque son divertidos, mientras que el $72 \%$ que respondió que los videojuegos son atractivos porque suponen un reto y un desafío vendría a corresponderse con las teorías de Suits (1976), Kelley (1988) y Salen y Zimmerman (2006), relativas a la importancia de la norma como elemento lúdico. Del mismo modo, otro de los aspectos planteados por Huizinga, como es la interacción, haría lo propio con las respuestas ofrecidas por el $42 \%$ de los encuestados, que afirman que los videojuegos les resultan atractivos porque suponen una manera de interactuar con familia y amigos.
} 
maneras de identificar una realidad y de taxonomizar los factores que la conforman.

Resulta especialmente interesante, tanto por relevancia como por profundidad, la reflexión de Prensky (2001) al respecto del componente lúdico en los videojuegos. En esta línea, y como complemento a la identificación de los motivos por los que los videojuegos ejercen un alto grado de atracción sobre el jugador, el autor reflexiona acerca de las características estructurales de estas herramientas.

1. A Clear Overall Vision. As in any other artistic endeavor, a clear vision is key to making a good game. [...] 2. A Constant Focus on the Player Experience. [...] 3. A Strong Structure. [...] Some game structures are fractal, with "convexities of convexities." But the structure must be thought out carefully up-front. [...] 4. Highly adaptive. The game must be able to be fun for a variety of players. [...] 5. Easy to learn, hard to master. The best games are often the ones that can be learned in only a few minutes, but provide hours or even lifetimes of challenge. [...] 6. Stays within the "flow state." A successful game needs to constantly walk that fine line between "not too hard" and "not too easy," and do it for a variety of players. [...] 7. Provides frequent rewards, not penalties. Rewards are an incentive to go on. [...] 8. Includes Exploration and Discovery. While not typically a part of puzzle or sports games, players like to "explore their turf," and uncover progressively various portions of the landscape. 9. Provides mutual assistance - thing helps solve another. [...] 10. Has an interface that is very useful. What is important for a successful game is not a simple interface, but a highly useful one. [...] 11. Includes the ability to save progress. (2001: 05-23) 
De igual manera, Fernández (2004) trata las características que han de encontrarse en un videojuego que pretenda captar y mantener la atención del jugador, realizando su análisis desde la perspectiva del desarrollo. De este modo, asegura que los videojuegos:

Diferencian claramente la interactividad de lo que no lo es.

[...] Deben promover que los jugadores hagan cosas, y no que éstas aparezcan sin su actuación. [...] Ocultan información al jugador, pero éstos reconocen lo que buscan en cuanto lo ven. [...] Han de mantener concentrado al jugador en todo momento. [...] Incitan a los videojugadores a pensar que siempre saldrán airosos, y esperan del videojuego que les ayude si en algún momento lo necesitan. [...] Nunca presentan situaciones de las que el jugador no pueda salir. [...] Deben proponer metas a corto plazo. [...] Promueven en los jugadores la idea de que las soluciones razonables que proponen sean las correctas, y que impere el sentido común en el videojuego. [...] No deben presentar las mismas pruebas una y otra vez. [...] Deben dar la oportunidad al jugador de salir y volver a entrar en el juego cuando este lo desee.

Volviendo al polo del jugador, Wood, Griffiths, Chappell y Davies (2004: 1), en su investigación acerca de las características estructurales de los videojuegos, identifican la importancia de elementos tales como el realismo, las posibilidades de personalización o la facilidad de hacerse con el manejo del juego:

Although there were major gender differences, one of the main overall findings was the importance of a high degree of realism (i.e., realistic sound, graphics, and settings). Other important characteristics included a rapid 
absorption rate, character development, the ability to customize the game, and multi-player features.

Por su parte, Gee identifica una serie de características que comparten los buenos videojuegos, afirmando que en realidad se tratan de principios idóneos en el marco de la enseñanza y clasificándolos además en tres categorías distintas: principios centrados en el jugador, en la resolución de problemas y en la comprensión de la semiótica del propio juego. En relación con las características centradas en el polo del jugador, Gee (2005: 6-9) enumera los siguientes principios:

Co-design. In good games, players feel that their actions and decisions $[\ldots]$ are co-creating the world they are in and the experiences they are having. [...]

Customize. [In good games] players are able to customize the game play to fit their learning and playing styles. [...]

Identity. Good games offer players identities that trigger a deep investment on the part of the player. [...]

Manipulation and Distributed Knowledge. The more and better a player can manipulate a character, the more the player invests in the game world. Good games offer characters that the player can move intricately, effectively, and easily through the world. $[\ldots]$

Well-ordered Problems: [...] in good games [...] early problems are designed to lead players to form good guesses about how to proceed when they face harder problems later on in the game. $[\ldots]$

Con respecto a los principios presentes en los buenos videojuegos relacionados con la resolución de problemas, Gee (2005: 10-13) trata aspectos 
como nivel de dificultad y feedback, práctica de habilidades, escenarios de práctica segura o desarrollo de habilidades:

Pleasantly Frustrating: Good games adjust challenges and give feedback in such a way that different players feel the game is challenging but doable and that their effort is paying off. [...] Cycles of Expertise: Good games create and support the cycle of expertise, with cycles of extended practice, tests of mastery of that practice, then a new challenge, and then new extended practice. $[\ldots]$

Information 'On Demand' and 'Just in Time': Good games give verbal information [...] 'just in time' and 'on demand' in a game. Players don't need to read a manual to start. [...]

Fish Tanks: Good games offer players fish tanks [simplified systems], either as tutorials or as their first level or two. [...]

Sandboxes: Good games offer players, either as tutorials or as their first level or two, sandboxes [safe havens]. In many good games, the first level is a sandbox or close to it. [...]

Skills as Strategies: In good games, players learn and practice skill packages as part and parcel of accomplishing things they need and want to accomplish. [...]

Finalmente, en relación a la semiótica del propio juego, Gee (2005: 14) identifica dos principios fundamentales: concepción del sistema y comprensión del significado a través de la acción. De este modo, afirma por una parte que los juegos permiten al jugador entender cada uno de los elementos dentro del propio juego, concibiendo las reglas en el marco de una tipología o género concreto. Por otra, señala que del mismo modo que las palabras y los conceptos articulan su significado en torno a la experiencia personal del individuo, los buenos videojuegos lo hacen en torno a las experiencias de juego, y no por medio de explicaciones teóricas: 
System Tbinking: People learn skills, strategies, and ideas best when they see how they fit into an overall larger system to which they give meaning. [...] Good games help players see and understand how each of the elements in the game fit into the overall system of the game and its genre (type). [...]

Meaning as Action Image: [...] games make the meanings of words and concepts clear through experiences the player has and activities the player carries out, not through lectures, talking heads, or generalities. [...]

Como se ha explicado en páginas anteriores, son muchos los motivos por los que los videojuegos resultan atractivos para el usuario: por una parte, logran captar y mantener la atención como consecuencia de la motivación que su uso despierta (Malone, 1981; Malone y Lepper, 1987). Otros autores inciden en que su dificultad representa un reto para el usuario, consiguiendo su implicación (Marquès, 2000), y en su carácter interactivo, frente a otras formas de ocio como la televisión o el cine, que exige una implicación activa por parte del jugador durante su desarrollo (Cortés, 2010). En otras ocasiones, para intentar explicar su atractivo se ha incidido en aspectos como el hecho de que ofrecen un feedback constante adaptado a los conocimientos del jugador o que aumentan su propio ego por medio de sistemas de recompensas (Fernández, 2004; Prensky, 2001).

Habida cuenta de todo ello, no es de extrañar que se plantee por su uso en otros entornos distintos a los tradicionales. De este modo, desde la evidencia de que captan la atención del jugador y logran mantenerla de forma más eficiente que otras herramientas, se han desarrollado una serie de videojuegos específicamente diseñados para propósitos distintos del mero entretenimiento. Tales videojuegos, enmarcados bajo la denominación de «juego serio», parten de una concepción peculiar respecto a la finalidad de la propia herramienta y cuentan con una serie de características que les diferencia de los videojuegos 
convencionales, entendidos, tal como se ha mencionado, como herramientas para el desarrollo de un «ejercicio lúdico delimitado por normas ejercido de forma voluntaria a través de un hardware específico» con las características que se les presuponen, descritas en el presente apartado.

\section{Videojuegos ¿serios?}

En palabras de Abt (1970), «a serious game is a game designed for a primary purpose other than pure entertainment», es decir, un juego cuya finalidad principal no es la de divertir al usuario. ¿Cuál es entonces el objetivo de los juegos serios? Zyda (2005: 26) afirma que, a diferencia de otros juegos que únicamente ofrecen entretenimiento, «serious games use pedagogy to infuse instruction into the game play experience»y los define como «a mental contest, played with a computer in accordance with specific rules, that uses entertainment to further government or corporate training, education, health, public policy, and strategic communication objectives».

Como se deduce de ello, los videojuegos serios se plantean principalmente el aprendizaje, relegando el aspecto lúdico a un segundo plano. En esta línea, encontramos la definición de Michael y Chen (2006: 17), quienes afirman que «a serious game is a game in which education (in its various forms) is the primary goal, rather than entertainment». No obstante, como se ha mencionado anteriormente, la totalidad de los razonamientos expuestos por los que tanto juego como videojuegos resultan atractivos confluyen en un motivo principal: proporcionan diversión al jugador. Retomando la definición propuesta al respecto de los videojuegos, «ejercicio lúdico delimitado por normas ejercido de forma voluntaria a través de un hardware específico», el concepto de «serio» contraviene la propia esencia del juego en cuanto ejercicio lúdico.

¿Puede un juego captar la atención del jugador y mantenerla sin ofrecerle específicamente diversión? ¿Puede un videojuego serio recibir consideración 
de juego si no es eminentemente lúdico? Michael y Chen (2006) inciden en que el hecho de que los juegos serios sean considerados como tal no implica que no sean divertidos o entretenidos, sino que únicamente existen otros aspectos formativos de mayor importancia. Sin embargo, los diseñadores de juegos profesionales no dudan en referir a la diversión como el aspecto central de un videojuego en torno al cual debe girar su desarrollo (Costikyan, 2002; Crawford, 1982; Koster, 2005; Schell, 2008), mientras que Marsh (2011: 61) afirma específicamente que «serious games challenges our understanding of generally accepted characteristics such as, challenge, play and fun, which are largely associated with and borrowed from video games».

Por otra parte, a partir de las definiciones propuestas por Huizinga (1949) y Callois (2006), entre otros, entendemos tanto el juego como los videojuegos como una actividad voluntaria: parece evidente, por tanto, que la actividad lúdica deja de serlo desde el momento en que es impuesta al jugador o desde el momento en que percibe que no es más que medio para alcanzar un objetivo distinto al de la mera diversión (cf. Wrzesien \& Alcañiz Raya, 2010). Sin embargo, como indican Susi, Johannesson y Backlund (2007: 5), resulta complicado concebir los «juegos serios» diseñados para transmitir contenidos docentes siendo utilizados voluntariamente en un contexto externo al aula: «serious games often violate voluntariness in that trainees may be ordered to play a particular game as part of their training». En esta línea, Leyland (1996) señala que «[...] video games with "educational" features have not fared well in the marketplace. The "educational" content tends to come at the expense of the gameplay and control is taken out of the hands of the player», mientras que Facer et al. (2003) apuntan que «edutainment titles have a strong educational component but often do not reflect the motivational drive of commercial titles». 


\section{Conclusiones}

Dado que los «juegos serios» apenas tienen incidencia en el mercado, y su uso parece estar confinado al contexto educativo o a circunstancias externas a la búsqueda de diversión por parte del jugador, resulta coherente plantearse la duda de si pueden recibir la consideración de «juegos» en línea con la definición de videojuego propuesta en páginas anteriores.

¿Significa esto que los videojuegos serios no son videojuegos? Como ya hemos indicado, si atendemos a sus características más notables (no prevalencia del componente lúdico en su desarrollo e incidencia no significativa de su uso fuera de entornos académicos controlados) y tomamos en consideración la definición propuesta para el concepto de videojuego a partir de otras definiciones anteriores, podemos afirmar que contravienen las características distintivas de diversión y voluntariedad, especialmente aquellos para la educación y la formación.

Por tanto, opinamos que, aunque los videojuegos serios reciban consideración de videojuegos, puesto que comparten las características relativas al soporte tecnológico sobre el que se asientan, se han de tener especialmente en cuenta cuantas circunstancias se puedan derivan, en materia investigadora, del hecho de que los primeros pasen por alto, en la mayoría de los casos, la esencia lúdica de éstos.

\section{Bibliografía}

Abt, C. C. (1970). Serious Games. New York: Viking Press.

Avedon, E. M. M., \& Sutton-Smith, B. (1981). The Study of Games. New York: John Wiley \& Sons, Inc. 
Buytendijk, F. J. J. (1933). Wesen und Sinn des Spiels. Das Spielen des Menschen und der Tiere als Erscheinungsform der Lebenstriebe. Berlin: Wolff.

Callois, R. (2006). The definition of play and the classification of games. In K. Salen \& E. Zimmerman (Eds.), The game design reader (pp. 122-155). MIT press.

Cortés Gómez, S. (2010). Mundos imaginarios y realidad virtual. Videojuegos en las aulas. Universidad de Alcalá. Departamento de Psicopedagogía y Educación Física, Universidad de Alcalá.

Costikyan, G. (2002). I have no words \& I must design: Toward a critical vocabulary for games. In Computer Games and Digital Cultures Conference Proceedings (pp. 9-33). Tampere: Tampere University Press.

Crawford, C. (1982). The art of computer game design. Berkeley, CA: McGrawHill/Osborne Media.

Evans, J., \& Pellegrini, A. (1997). Surplus Energy Theory: an enduring but inadequate justification for school break-time. Cational Review, 49(3), 229-236.

Facer, K., Furlong, J., Furlong, R., \& Sutherland, R. (2003). "Edutainment" software: a site for cultures in conflict. In R. Sutherland, G. Claxton, \& A. Pollard (Eds.), Learning and Teaching Where Worldviews meet. London: Trentham Books.

Fernández Lobo, I. (2004). Herramientas para la creación de videojuegos. Comunicación Y Pedagogía: Nuevas Tecnologías Y Recursos Didácticos, (199), 71-77.

Frasca, G. (2001). Videogames of the oppressed: Videogames as a means for critical thinking and debate. Georgia Institute of Technology. 
Freud, S. (1920). Jenseits des Lustprinæips. Wien: Internationaler Psychoanalytischer Verlag.

Gallego Durán, F. J., \& Llorens Largo, F. (2011). Qué nos enseña Pacman? Lecciones aprendidas desarrollando videojuegos educativos. In I Congreso Internacional sobre Aprendizaje, Innovación y Competitividad (CINAIC 2011), Madrid, 26-28 septiembre 2011. Retrieved from http://hdl.handle.net/10045/19013

Garaigordobil Landazábal, M. T. (2007). La teoría del origen histórico-social del juego de Elkonin en la investigación del juego infantil. In-Fan-Cia: Educar de 0 a 6 Años. Revista de La Associació de Mestres Rosa Sensat, (102), 3-7.

García Gigante, B. (2009). Videojuegos: Medio de ocio, cultura popular y recurso didáctico para la enseñanza y aprendizaje de las matemáticas escolares. Universidad Autónoma de Madrid.

Gee, J. P. (2005). Learning by design: Good video games as learning machines. E-Learning and Digital Media, 2(1), 5-16.

Gutiérrez Delgado, M. (2004). La bondad del juego, pero... EA, Escuela Abierta: Revista de Investigación Educativa, (7), 153-182.

Hall, G. S. (1906). Youth: Its Education, Regiment, and Hygiene. New York: Appleton.

Huizinga, J. (1949). Homo Ludens: A Study of the Play-Element in Culture. Routledge \& Kegan Paul Ltd. 
Juul, J. (2003). The Game, the Player, the World: Looking for a Heart of Gameness. In Level Up: Digital Games Research Conference Proceedings (pp. 30-45). Utrech: Utrech University.

Kelley, D. (1988). The art of reasoning. New York: W. W. Norton \& Company.

Kirriemuir, J. (2002). Video gaming, education and digital learning technologies: Relevance and opportunities. D-Lib Magazine, 8(2).

Kirriemuir, J., \& McFarlane, A. (2004). Literature Review in Games and Learning. Future Lab Series Report, 8(2), 39. http://doi.org/10.1111/j.15410072.1974.tb01308.x

Koster, R. (2005). A Theory of Fun for Game Design. Scottsdale, Arizona: Paraglyph Press, Inc.

Lazarus, M. (1883). Über die Reize des Spiels. Berlin: Dümmlers Verlagsbuchhandlung.

Levis, D. (2005). Videojuegos y alfabetización digital. Aula de Innovación Educativa, 147.

Leyland, B. (1996). How can computer games offer deep learning and still be fun? In A. S. for Computers in Learning in Tertiary Education, A. Christie, P. James, \& B. Vaughan (Eds.), ASCILITE '96: Making new connections. Proceedings of the 13th Annual Conference of the Australian Society for Computers in Learning in Tertiary Education.

Lin, S., \& Lepper, M. R. R. (1987). Correlates of children's usage of videogames and computers. Journal of Applied Social Psychology, 17(1), 72-93. 
Malone, T. W. (1981). Toward a theory of intrinsically motivating instruction. Cognitive Science, 5(4), 333-369.

Malone, T. W., \& Lepper, M. R. (1987). Making Learning Fun: A Taxonomy of Intrinsic Motivations for Learning. In R. E. Snow \& M. J. Farr (Eds.), Aptitude, Learning, and Instruction. Volume 3: Cognitive and Affective Process Analyses. Hillsdale, N.J.: Erlbaum.

Marquès Graells, P. (2000). Los videojuegos y sus posibilidades educativas. Retrieved from http://peremarques.pangea.org/pravj.htm

Marsh, T. (2011). Serious games continuum: Between games for purpose and experiential environments for purpose. Entertainment Computing, 2(2), 61-68. http://doi.org/10.1016/j.entcom.2010.12.004

Martínez Rodríguez, E. (2008). El juego como escuela de vida: Karl Groos. MAGISTER. Revista Miscelánea de Investigación, (22), 7-22.

Méndez González, R., \& Calvo-Ferrer, J. R. (2017). Videojuegos y [para]traducción: Aproximación a la práctica localizadora. Granada: Comares.

Michael, D., \& Chen, S. (2006). Serious games: Games that educate, train, and inform. Boston, MA: Thomson Course Technology.

Montañés Rodríguez, J., Parra, M., Sánchez, T., Sánchez, M. J., López, R., Latorre Postigo, J. M.,

Turégano, P. (2000). El juego en el medio escolar. Ensayos: Revista de La Facultad de Educación de Albacete, (15), 235-260. 
Pelegrina del Río, M., \& Tejeiro Salguero, R. (2003). Los videojuegos: Qué son y cómo nos afectan. Barcelona: Ariel.

Piaget, J. (1945). Play, dreams and imitation in childhood. London: Heinemann.

Prensky, M. (2001). Digital Game-Based Learning. New York: McGraw-Hill.

Provenzo, E. F. F. (1991). Video Kids: Making Sense of Nintendo. Cambridge, MA: Harvard University Press.

Robbins, D. (2001). Vygotsky's Psychology-Philosophy: A Metaphor for Language Theory and Learning. New York: Kluwer Academic.

Rodríguez, E. (Ed.). (2002). Jóvenes y Videojuegos: Espacio, significación y conflictos. Madrid: FAD.

Romero, V., \& Gómez, M. (2008). El juego infantil y su metodología. Barcelona: Altamar.

Salen, K., \& Zimmerman, E. (2004). The rules of play: Game design fundamentals. Cambridge, MA: MIT press.

Salen, K., \& Zimmerman, E. (2006). The game design reader. Cambridge, MA: MIT press.

Salen, K., Zimmerman, E., \& Goldstein, J. (2005). Game Designe and Meaningful Play. In J. Raessens (Ed.), Handbook computer game studies. Cambridge, MA: MIT press. 
Schell, J. (2008). The Art of Game Design: A book of lenses. Burlington, Massachusetts: Morgan Kaufmann.

Schiller, F. (1795). Briefe über die ästhetische Eræiehung des Menschen. Retrieved from http://www.dreigliederung.de/gliederung/schillersbriefe.html

Suits, B. (1976). The grasshopper: Games, life and utopia. Toronto: University of Toronto Press.

Susi, T., Johannesson, M., \& Backlund, P. (2007). Serious games. An overview. Skövde: University of Skövde.

Tabernero del Río, S. M. (1997). La educación funcional de E. Claparède. Revista de Pedagogía de La Universidad de Salamanca, (9), 45-72.

Tavinor, G. (2009). The Definition of Videogames Revisited. In The Philosophy of Computer Games Conference. Oslo.

Winnicott, D. (1982). Playing and Reality. London: Routledge.

Wood, R. T. A., Griffiths, M. D., Chappell, D., \& Davies, M. N. O. (2004). The structural characteristics of video games: a psycho-structural analysis. Cyberpsychology and Behavior, 7(1), 1-10.

Wrzesien, M., \& Alcañiz Raya, M. (2010). Learning in serious virtual worlds: Evaluation of learning effectiveness and appeal to students in the E-Junior project. Computers \& Education, 55(1), 178-187. http:/ / doi.org/10.1016/j.compedu.2010.01.003

Zyda, M. (2005). From Visual Simulation to Virtual Reality to Games. Computer, 38(9), 25-32. 


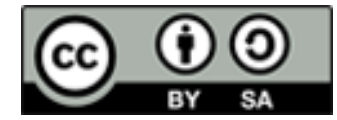

Licencia Creative Commons

Miguel Hernández Communication Journal mhjournal.org

\section{Cómo citar este texto:}

José Ramón Calvo-Ferrer (2018) Juegos, videojuegos y juegos serios: Análisis de los factores que favorecen la diversión del jugador. Miguel Hernández Communication Journal, 9 (1), pp. 191 a 226. Universidad Miguel Hernández, UMH (Elche-Alicante).

DOI: http://dx.doi.org/10.21134/mhcj.v0i9.232 